\title{
Performance Analysis of Space-Time Coding With Imperfect Channel Estimation
}

\author{
Parul Garg, Ranjan K. Mallik, Senior Member, IEEE, and Hari M. Gupta
}

\begin{abstract}
We analyze the error performance of a space-time coding system using $N$ transmit and $M$ receive antennas with imperfect channel estimation in flat Rayleigh fading. A least-squares estimate of the channel matrix is obtained by using a sequence of pilot code vectors. The estimate is found to be perturbed by an $M \times N$ perturbation matrix with zero-mean circular Gaussian entries. Using the characteristic function of the decision variable, we derive a closed-form expression for the pairwise error probability (PEP). From the same expression, the PEP in case of perfect channel estimation is also obtained. Numerical results show the degradation in performance due to imperfect channel estimation that can be compensated by increasing the number of receive antennas.
\end{abstract}

Index Terms-Imperfect channel estimation, pairwise error probability, pilot code vectors, Rayleigh fading, space-time coding.

\section{INTRODUCTION}

$\mathbf{S}$ PACE-TIME coding [1], [2], which uses the advantage of transmitter diversity, is an effective technique to improve the performance of wireless communication systems. In spacetime coding, different signals are simultaneously transmitted from different transmit antennas. The signal which is received is the superposition of the different transmitted signals, and the detection process needs estimates of the channel parameters [3]. Performance analysis methods often consider perfect channel estimates under the assumption that the estimation errors are negligible. Although perfect estimates are desirable, channel estimation methods used in practice give rise to imperfections [4], [5]. It is therefore of considerable relevance to study the effect of imperfect channel estimation on performance. One convenient method of estimation of channel parameters in a space-time coding system is by using pilot code sequences. Owing to the presence of additive noise in the received signal, we apply in this paper a least-squares based channel estimation technique to a space-time coding system using multiple transmit and receive antennas in flat Rayleigh fading and analyze its error performance. The estimate of the channel matrix is obtained from a sequence of pilot code vectors. Using the characteristic func-

Manuscript received February 18, 2003; revised June 14, 2003, September 1, 2003; accepted October 27, 2003. The editor coordinating the review of this paper and approving it for publication is $\mathrm{H}$. Li. This paper was presented in part at the IEEE International Conference on Personal Wireless Communications, New Delhi, India, December 15-17, 2002.

The authors are with the Department of Electrical Engineering, Indian Institute of Technology, Delhi, Hauz Khas, New Delhi 110016, India (e-mail: parul_saini@yahoo.co.in; rkmallik@ee.iitd.ernet.in; hmgupta@ee.iitd.ernet.in).

Digital Object Identifier 10.1109/TWC.2004.840202 tion (cf.) of the decision variable, we derive a closed-form expression for the pairwise error probability (PEP). From the same expression, the PEP in case of perfect channel estimation is also obtained. We apply our result to an example of a system using the Alamouti code [2] with binary phase-shift keying (BPSK).

The paper is organized as follows. Section II gives the basic model for a wireless communication system employing spacetime coding. We then present the channel estimation technique in Section III. Section IV discusses the performance criterion with imperfect channel estimation. Section V analyzes the error performance in terms of the PEP. As an example, the performance of a system using the Alamouti code with two transmit antennas and BPSK for imperfect as well as perfect channel estimation is presented in Section VI. Section VII gives some concluding remarks.

\section{MODEL}

Consider a communication system that employs space-time coding [1] with $N$ transmit and $M$ receive antennas. At time index $l$, the space-time encoder encodes the information symbol $s(l)$ into $N$ code symbols $c_{1}(l), c_{2}(l), \ldots, c_{N}(l)$, which are transmitted by the $N$ antennas at the same time.

The complex baseband signal received at time index $l$ by the $i$ th antenna after matched filtering is given by [1], [6]

$$
r_{i}(l)=\sqrt{2 E_{s}} \sum_{j=1}^{N} h_{i j}(l) c_{j}(l)+n_{i}(l), \quad i=1, \ldots, M
$$

where $2 E_{s}$ is the average energy of the baseband signal constellation, $h_{i j}$ the complex fading channel gain from the $j$ th transmit antenna to the $i$ th receive antenna, and $n_{i}(l)$ the additive white Gaussian noise with power spectral density $2 N_{0}$. Since the noise is white, the noise samples for different time indexes, denoted as $\left\{n_{i}(l)\right\}$, are independent and identically distributed (i.i.d.) zero-mean complex circular Gaussian random variables, each having a $\mathcal{C N}\left(0,2 N_{0}\right)$ distribution. We also assume that $n_{i}(l)$ and $n_{k}(l)$ are independent for $i \neq k, 1 \leq i, k \leq$ $M$.

The $N \times 1$ code vector transmitted from the $N$ antennas at time index $l$ is denoted as

$$
\mathbf{c}_{l}=\left[c_{1}(l) \quad c_{2}(l), \ldots, c_{N}(l)\right]^{T}
$$

where $(\cdot)^{T}$ denotes transpose, and the corresponding $M \times 1$ channel vector from the $j$ th transmit antenna to the $M$ receive antennas as

$$
\mathbf{h}_{j}(l)=\left[h_{1 j}(l) \quad h_{2 j}(l), \ldots, h_{M j}(l)\right]^{T} .
$$


In addition, let

$$
\mathbf{n}(l)=\left[n_{1}(l) \quad n_{2}(l), \ldots, n_{M}(l)\right]^{T}
$$

be the $M \times 1$ noise vector at the receive antennas. Note that the noise vectors for different time indexes, represented as $\{\mathbf{n}(l)\}$, are i.i.d. complex circular Gaussian random vectors, each $\mathbf{n}(l)$ having a $\mathcal{C N}\left(\mathbf{0}_{M \times 1}, 2 N_{0} \mathbf{I}_{M}\right)$ distribution, where $\mathbf{I}_{M}$ denotes the $M \times M$ identity matrix.

The $M \times N$ channel matrix $\mathbf{H}(l)$, which is independent of the noise, is given by $\mathbf{H}(l)=\left[\mathbf{h}_{1}(l), \mathbf{h}_{2}(l), \ldots, \mathbf{h}_{N}(l)\right]$, and the $M \times 1$ received signal vector $\mathbf{r}(l)$ by $\mathbf{r}(l)=$ $\left[r_{1}(l), \quad r_{2}(l), \ldots, r_{M}(l)\right]^{T}$. We can therefore rewrite (1) in matrix form as [1]

$$
\mathbf{r}(l)=\sqrt{2 E_{s}} \mathbf{H}(l) \mathbf{c}_{l}+\mathbf{n}(l) .
$$

Owing to flat fading, the channel matrix $\mathbf{H}(l)$ is assumed to be constant over the time indexes which span the pilot transmission phase followed by the encoded data transmission phase. Hence, we denote the channel matrix as

$$
\mathbf{H}=\left[\begin{array}{ll}
\mathbf{h}_{1} & \mathbf{h}_{2}, \ldots, \mathbf{h}_{N}
\end{array}\right] .
$$

Further, we consider a Rayleigh fading channel, with entries of the channel matrix $\mathbf{H}$ being i.i.d. zero-mean complex circular Gaussian random variables each having a $\mathcal{C N}(0, \Omega)$ distribution.

\section{Channel Estimation}

We use a sequence of $L_{p}$ pilot code vectors $\mathbf{c}_{p_{1}}, \ldots, \mathbf{c}_{p_{L_{p}}}$ which form the $N \times L_{p}$ pilot code matrix $\mathbf{C}_{p}$ given by

$$
\mathbf{C}_{p}=\left[\begin{array}{ll}
\mathbf{c}_{p_{1}} & \mathbf{c}_{p_{2}}, \ldots, \mathbf{c}_{p_{L_{p}}}
\end{array}\right] .
$$

When the pilot code matrix is transmitted, we receive

$$
\mathbf{r}_{p}(l)=\sqrt{2 E_{s}} \mathbf{H} \mathbf{c}_{p_{l}}+\mathbf{n}_{p}(l), \quad l=1, \ldots, L_{p}
$$

where $\mathbf{n}_{p}(1), \ldots, \mathbf{n}_{p}\left(L_{p}\right)$ are i.i.d. complex circular Gaussian random vectors, each having a $\mathcal{C N}\left(\mathbf{0}_{M \times 1}, 2 N_{0} \mathbf{I}_{M}\right)$ distribution.

Let the received pilot signal matrix $\mathbf{R}_{p}$ and the pilot noise matrix $\mathbf{N}_{p}$ be given by

$$
\begin{aligned}
\mathbf{R}_{p} & =\left[\mathbf{r}_{p}(1), \quad \mathbf{r}_{p}(2), \ldots, \mathbf{r}_{p}\left(L_{p}\right)\right] \\
\mathbf{N}_{p} & =\left[\mathbf{n}_{p}(1), \quad \mathbf{n}_{p}(2), \ldots, \mathbf{n}_{p}\left(L_{p}\right)\right] .
\end{aligned}
$$

Using (9) and (10), we can rewrite (8) as

$$
\mathbf{R}_{p}=\sqrt{2 E_{s}} \mathbf{H C}_{p}+\mathbf{N}_{p} .
$$

From (11), we obtain a least-squares estimate of the channel matrix [4], [5] which is given by

$$
\hat{\mathbf{H}}=\frac{1}{\sqrt{2 E_{s}}} \mathbf{R}_{p} \mathbf{C}_{p}^{H}\left(\mathbf{C}_{p} \mathbf{C}_{p}^{H}\right)^{-1}
$$

where $(\cdot)^{H}$ denotes the Hermitian (conjugate transpose) operator. Note that we need to choose $\mathbf{C}_{p}$ such that $\mathbf{C}_{p} \mathbf{C}_{p}^{H}$ is invertible, which implies that $L_{p} \geq N$. Combining (12) and (11), we get

$$
\hat{\mathbf{H}}=\mathbf{H}+\frac{1}{\sqrt{2 E_{s}}} \mathbf{N}_{p} \mathbf{C}_{p}^{H}\left(\mathbf{C}_{p} \mathbf{C}_{p}^{H}\right)^{-1} .
$$

Note that the estimate of $\mathbf{H}$ is perturbed by an $M \times N$ perturbation matrix with zero-mean circular Gaussian entries, which are uncorrelated if $\mathbf{C}_{p}$ is chosen such that $\mathbf{C}_{p} \mathbf{C}_{p}^{H}$ is a scaled version of $\mathbf{I}_{N}$, and correlated otherwise.

\section{Performance CRiterion}

Let $\mathcal{C}_{N, L}$ denote the set of $N \times L$ code matrices used for transmission. Suppose that the code matrix

$$
\mathbf{C}=\left[\begin{array}{ll}
\mathbf{c}_{1} & \mathbf{c}_{2}, \ldots, \mathbf{c}_{L}
\end{array}\right]
$$

is transmitted after the channel has been estimated. At the receiver, we choose the code matrix

$$
\tilde{\mathbf{C}}=\left[\begin{array}{ll}
\tilde{\mathbf{c}}_{1} & \tilde{\mathbf{c}}_{2}, \ldots, \tilde{\mathbf{c}}_{L}
\end{array}\right]
$$

using the minimum distance rule, which results in

$$
\tilde{\mathbf{C}}=\arg \left\{\min _{\left[\mathbf{x}_{1}, \ldots, \mathbf{x}_{L}\right] \in \mathcal{C}_{N, L}} \sum_{l=1}^{L}\left\|\mathbf{r}(l)-\sqrt{2 E_{s}} \hat{\mathbf{H}} \mathbf{x}_{l}\right\|^{2}\right\}
$$

where $\|\cdot\|$ denotes the $L_{2}$-norm or Euclidean norm of a vector. Substituting (5) and (13) in (16), we get the PEP, which is given by

$$
\begin{aligned}
P(\mathbf{C} & \rightarrow \tilde{\mathbf{C}}) \\
= & \operatorname{Pr}\left\{\sum_{l=1}^{L} \| \sqrt{2 E_{s}} \mathbf{H}\left(\mathbf{c}_{l}-\tilde{\mathbf{c}}_{l}\right)\right. \\
& +\mathbf{n}(l)-\mathbf{N}_{p} \mathbf{C}_{p}^{H}\left(\mathbf{C}_{p} \mathbf{C}_{p}^{H}\right)^{-1} \tilde{\mathbf{c}}_{l} \|^{2} \\
& \left.<\sum_{l=1}^{L}\left\|\mathbf{n}(l)-\mathbf{N}_{p} \mathbf{C}_{p}^{H}\left(\mathbf{C}_{p} \mathbf{C}_{p}^{H}\right)^{-1} \mathbf{c}_{l}\right\|^{2}\right\}
\end{aligned}
$$

where the $M \times 1$ complex Gaussian vectors $\mathbf{n}(1), \ldots, \mathbf{n}(L)$ are independent of the $M \times L_{p}$ complex Gaussian matrix $\mathbf{N}_{p}$.

When the channel estimate is perfect, we have $\hat{\mathbf{H}}=\mathbf{H}$, and the PEP is given by

$$
\begin{aligned}
& P_{\text {per }} \mathrm{f}(\mathbf{C} \rightarrow \tilde{\mathbf{C}}) \\
& \quad=\operatorname{Pr}\left\{\sum_{l=1}^{L}\left\|\sqrt{2 E_{s}} \mathbf{H}\left(\mathbf{c}_{l}-\tilde{\mathbf{c}}_{l}\right)+\mathbf{n}(l)\right\|^{2}<\sum_{l=1}^{L}\|\mathbf{n}(l)\|^{2}\right\} .
\end{aligned}
$$


From the Gaussian distribution of $\mathbf{n}(l)$, it can be easily shown that the conditional PEP, conditioned on the channel matrix $\mathbf{H}$, is given by

$$
P_{\text {per }} \mathrm{f}(\mathbf{C} \rightarrow \tilde{\mathbf{C}} \mid \mathbf{H})=Q\left(\sqrt{\frac{E_{s}}{2 N_{0}} \sum_{l=1}^{L}\left\|\mathbf{H}\left(\mathbf{c}_{l}-\tilde{\mathbf{c}}_{l}\right)\right\|^{2}}\right)
$$

where $Q(\cdot)$ denotes the Gaussian- $Q$ function. The PEP can be obtained by averaging the conditional PEP over the statistics of $\mathbf{H}$, as in [7].

When the channel has an imperfect estimate given by (13), we can write

$$
\hat{\mathbf{H}}=\mathbf{H}+\Delta \mathbf{H}
$$

where the perturbation matrix $\Delta \mathbf{H}$ is given by

$$
\Delta \mathbf{H}=\frac{1}{\sqrt{2 E_{s}}} \mathbf{N}_{p} \mathbf{C}_{p}^{H}\left(\mathbf{C}_{p} \mathbf{C}_{p}^{H}\right)^{-1}
$$

Denoting the random vector $\hat{\mathbf{n}}(l)$ as

$$
\hat{\mathbf{n}}(l)=\mathbf{n}(l)-\sqrt{2 E_{s}}(\Delta \mathbf{H}) \mathbf{c}_{l}
$$

we can express (17) in the form

$$
\begin{aligned}
P(\mathbf{C} & \rightarrow \tilde{\mathbf{C}}) \\
= & \operatorname{Pr}\left\{\sum_{l=1}^{L}\left\|\sqrt{2 E_{s}} \hat{\mathbf{H}}\left(\mathbf{c}_{l}-\tilde{\mathbf{c}}_{l}\right)+\hat{\mathbf{n}}(l)\right\|^{2}\right. \\
& \left.<\sum_{l=1}^{L}\|\hat{\mathbf{n}}(l)\|^{2}\right\}
\end{aligned}
$$

which can be simplified to yield

$$
\begin{aligned}
P(\mathbf{C} & \rightarrow \tilde{\mathbf{C}}) \\
= & \operatorname{Pr}\left\{2 \sqrt{2 E_{s}} \operatorname{Re}\left\{\sum_{l=1}^{L}\left[\hat{\mathbf{H}}\left(\mathbf{c}_{l}-\tilde{\mathbf{c}}_{l}\right)\right]^{H} \hat{\mathbf{n}}(l)\right\}\right. \\
& \left.<-2 E_{s} \sum_{l=1}^{L}\left\|\hat{\mathbf{H}}\left(\mathbf{c}_{l}-\tilde{\mathbf{c}}_{l}\right)\right\|^{2}\right\} .
\end{aligned}
$$

When the channel estimate is perfect, we have $\Delta \mathbf{H}=\mathbf{0}_{M \times N}$, resulting in

$$
\hat{\mathbf{H}}=\mathbf{H}, \quad \hat{\mathbf{n}}(l)=\mathbf{n}(l)
$$

and (23) reduces to (18). When we substitute (25) in (24), and find the conditional PEP, conditioned on $\mathbf{H}$, we obtain (19), because $\mathbf{H}$ and $\{\mathbf{n}(l)\}$ are independent. On the other hand, when the channel estimate is imperfect, although $\mathbf{H},\{\mathbf{n}(l)\}$, and $\Delta \mathbf{H}$ are independent, we see from (20) and (22) that both $\hat{\mathbf{H}}$ and $\{\hat{\mathbf{n}}(l)\}$ depend on $\Delta \mathbf{H}$, and are therefore, not independent. We can choose appropriate space-time codes such that the marginal statistics of $\hat{\mathbf{H}}$ becomes the same as the statistics of $\mathbf{H}$ and the marginal statistics of $\{\hat{\mathbf{n}}(l)\}$ becomes the same as the statistics of $\{\mathbf{n}(l)\}$. However, owing to the dependence on $\Delta \mathbf{H}$, the joint statistics of $\hat{\mathbf{H}}$ and $\{\hat{\mathbf{n}}(l)\}$ is not the same as the joint statistics of $\mathbf{H}$ and $\{\mathbf{n}(l)\}$. As a result, none of $P(\mathbf{C} \rightarrow \tilde{\mathbf{C}} \mid \mathbf{H}), P(\mathbf{C} \rightarrow$ $\tilde{\mathbf{C}} \mid \hat{\mathbf{H}})$, or $P(\mathbf{C} \rightarrow \tilde{\mathbf{C}} \mid \mathbf{H}, \Delta \mathbf{H})$, will have a form similar to (19). This calls for an alternative analysis of the PEP.

\section{Performance AnAlysis}

Instead of obtaining first the conditional PEP when the channel estimate is given by (13) and then averaging it over the statistics of $\mathbf{H}$ to get the PEP, we will derive an expression for the PEP given by (17) from the cf. of the decision variable $\mathcal{D}$ which is defined as

$$
\begin{aligned}
\mathcal{D} \triangleq & \sum_{l=1}^{L} \| \sqrt{2 E_{s}} \mathbf{H}\left(\mathbf{c}_{l}-\tilde{\mathbf{c}}_{l}\right) \\
& +\mathbf{n}(l)-\mathbf{N}_{p} \mathbf{C}_{p}^{H}\left(\mathbf{C}_{p} \mathbf{C}_{p}^{H}\right)^{-1} \tilde{\mathbf{c}}_{l} \|^{2} \\
& -\sum_{l=1}^{L}\left\|\mathbf{n}(l)-\mathbf{N}_{p} \mathbf{C}_{p}^{H}\left(\mathbf{C}_{p} \mathbf{C}_{p}^{H}\right)^{-1} \mathbf{c}_{l}\right\|^{2} .
\end{aligned}
$$

Define $M \times 1$ random vectors $\mathbf{a}_{l}, \tilde{\mathbf{a}}_{l}$, and $\mathbf{g}_{l}$ as

$$
\begin{aligned}
& \mathbf{a}_{l} \triangleq \mathbf{n}(l)-\mathbf{N}_{p} \mathbf{C}_{p}^{H}\left(\mathbf{C}_{p} \mathbf{C}_{p}^{H}\right)^{-1} \mathbf{c}_{l} \\
& \tilde{\mathbf{a}}_{l} \triangleq \mathbf{n}(l)-\mathbf{N}_{p} \mathbf{C}_{p}^{H}\left(\mathbf{C}_{p} \mathbf{C}_{p}^{H}\right)^{-1} \tilde{\mathbf{c}}_{l} \\
& \mathbf{g}_{l} \triangleq \sqrt{2 E_{s}} \mathbf{H}\left(\mathbf{c}_{l}-\tilde{\mathbf{c}}_{l}\right) .
\end{aligned}
$$

Further, define $L_{p} \times L$ matrices $\mathbf{B}$ and $\tilde{\mathbf{B}}$ as

$$
\begin{aligned}
& \mathbf{B} \triangleq \mathbf{C}_{p}^{H}\left(\mathbf{C}_{p} \mathbf{C}_{p}^{H}\right)^{-1} \mathbf{C} \\
& \tilde{\mathbf{B}} \triangleq \mathbf{C}_{p}^{H}\left(\mathbf{C}_{p} \mathbf{C}_{p}^{H}\right)^{-1} \tilde{\mathbf{C}} .
\end{aligned}
$$

From (27), we can rewrite the decision variable $\mathcal{D}$ in (26) as

$$
\mathcal{D}=\sum_{l=1}^{L}\left[\left\|\mathbf{g}_{l}+\tilde{\mathbf{a}}_{l}\right\|^{2}-\left\|\mathbf{a}_{l}\right\|^{2}\right] .
$$

The vectors $\mathbf{g}_{1}, \ldots, \mathbf{g}_{L}, \tilde{\mathbf{a}}_{1}, \ldots, \tilde{\mathbf{a}}_{L}, \mathbf{a}_{1}, \ldots, \mathbf{a}_{L}$ are jointly complex Gaussian. Define the $2 M L \times 1$ composite vector $\mathbf{v}$ as

$$
\mathbf{v} \triangleq\left[\begin{array}{c}
\mathbf{g}_{1}+\tilde{\mathbf{a}}_{1} \\
\vdots \\
\mathbf{g}_{L}+\tilde{\mathbf{a}}_{L} \\
\mathbf{a}_{1} \\
\vdots \\
\mathbf{a}_{L}
\end{array}\right]
$$

From the Gaussian statistics of $\mathbf{n}(1), \ldots, \mathbf{n}(L), \mathbf{N}_{p}$, and $\mathbf{H}$, we find that $\mathbf{v}$ is a zero-mean complex circular Gaussian random 
vector having a $\mathcal{C N}\left(\mathbf{0}_{2 M L \times 1}, \mathbf{K}_{v}\right)$ distribution, where the covariance matrix $\mathbf{K}_{v}$ is given by

$\mathbf{K}_{v}=2 N_{0}\left[\begin{array}{cc}\left(\mathbf{I}_{L}+\tilde{\mathbf{B}}^{T} \tilde{\mathbf{B}}^{*}\right) & \left(\mathbf{I}_{L}+\tilde{\mathbf{B}}^{T} \mathbf{B}^{*}\right) \\ +\frac{\Omega E_{s}}{N_{0}}(\mathbf{C}-\tilde{\mathbf{C}})^{T}(\mathbf{C}-\tilde{\mathbf{C}})^{*} & \left(\mathbf{I}_{L}+\mathbf{B}^{T} \mathbf{B}^{*}\right) \\ \left(\mathbf{I}_{L}+\mathbf{B}^{T} \tilde{\mathbf{B}}^{*}\right) & \otimes \mathbf{I}_{M}\end{array}\right]$

$\otimes$ denoting the Kronecker product, and $(\cdot)^{*}$ denoting complex conjugation. The decision variable $\mathcal{D}$ in (29) can be written as

$$
\mathcal{D}=\mathbf{v}^{H}\left[\begin{array}{cc}
\mathbf{I}_{M L} & \mathbf{0} \\
\mathbf{0} & -\mathbf{I}_{M L}
\end{array}\right] \mathbf{v}
$$

which is a Hermitian quadratic form in complex Gaussian random variables. Using the result of [8], we can express the cf. of $\mathcal{D}$ as

$$
\begin{aligned}
\Psi_{\mathcal{D}}(\jmath \omega) & =\mathbf{E}\left[e^{\jmath \omega \mathcal{D}}\right] \\
& =\frac{1}{\operatorname{det}\left(\mathbf{I}_{2 M L}-\jmath \omega\left[\begin{array}{cc}
\mathbf{I}_{M L} & \mathbf{0} \\
\mathbf{0} & -\mathbf{I}_{M L}
\end{array}\right] \mathbf{K}_{v}\right)} .
\end{aligned}
$$

Substituting (31) in (33), we get

$$
\Psi_{\mathcal{D}}(\jmath \omega)=\frac{1}{\left[\operatorname{det}\left(\mathbf{I}_{2 L}-2 \jmath \omega N_{0} \mathbf{A}\right)\right]^{M}}
$$

where $\mathbf{A}$ is a $2 L \times 2 L$ matrix given by

$$
\mathbf{A}=\left[\begin{array}{cc}
\left(\mathbf{I}_{L}+\tilde{\mathbf{B}}^{T} \tilde{\mathbf{B}}^{*}\right) & \left(\mathbf{I}_{L}+\tilde{\mathbf{B}}^{T} \mathbf{B}^{*}\right) \\
+\frac{\Omega E_{s}}{N_{0}}(\mathbf{C}-\tilde{\mathbf{C}})^{T}(\mathbf{C}-\tilde{\mathbf{C}})^{*} & \\
-\left(\mathbf{I}_{L}+\mathbf{B}^{T} \tilde{\mathbf{B}}^{*}\right) & -\left(\mathbf{I}_{L}+\mathbf{B}^{T} \mathbf{B}^{*}\right)
\end{array}\right]
$$

If $\Lambda_{1}, \ldots, \Lambda_{2 L}$ are the $2 L$ eigenvalues of $\mathbf{A}$, then the cf. of $\mathcal{D}$ can be simply written as

$$
\Psi_{\mathcal{D}}(\jmath \omega)=\frac{1}{\prod_{i=1}^{2 L}\left(1-2 \jmath \omega N_{0} \Lambda_{i}\right)^{M}} .
$$

The PEP is given by

$$
P(\mathbf{C} \rightarrow \tilde{\mathbf{C}})=\operatorname{Pr}(\mathcal{D}<0) .
$$

We now obtain the PEP from the cf. of $\mathcal{D}$ using the inversion theorem [9]. After changing the variable $\jmath \omega$ to $z=2 \jmath \omega N_{0}$ in (36), we get [9]

$$
\begin{aligned}
P(\mathbf{C} \rightarrow \tilde{\mathbf{C}})=- & {\left[\text { sum of residues of } \frac{\Psi_{\mathcal{D}}\left(\frac{z}{2 N_{0}}\right)}{z}\right.} \\
& \text { at poles on left-half } z \text {-plane }] .
\end{aligned}
$$

Note from (36) that the poles of $\Psi_{\mathcal{D}}\left[z /\left(2 N_{0}\right)\right]$ are given by $z=$ $1 / \Lambda_{i}, i=1, \ldots, 2 L$, all of which may not be distinct. Some of these poles will be on the left half $z$-plane. Let $\mu_{k}$ be a pole of order $p_{k}$. The PEP can then be expressed using (38) as

$$
\begin{aligned}
P(\mathbf{C} \rightarrow \tilde{\mathbf{C}})=- & \sum_{\begin{array}{c}
\text { pole } \mu_{k} \\
\text { of order } p_{k} \\
\text { such that } \\
\operatorname{Re}\left(\mu_{k}\right)<0
\end{array}} \frac{1}{\left(p_{k}-1\right) !} \frac{d^{p_{k}-1}}{d z^{p_{k}-1}} \\
& {\left.\left[\left(z-\mu_{k}\right)^{p_{k}} \frac{\Psi_{\mathcal{D}}\left(\frac{z}{2 N_{0}}\right)}{z}\right]\right|_{z=\mu_{k}} . }
\end{aligned}
$$

Since $\left(z-\mu_{k}\right)^{p_{k}}\left(\Psi_{\mathcal{D}}\left(z /\left(2 N_{0}\right)\right)\right) / z$ is a rational function in $z$, its $\left(p_{k}-1\right)$ th derivative can be conveniently obtained using Faa di Bruno's formula [10]. Thus, (39) provides us with a means of computing the PEP without the use of any integral.

We now consider the case when we use orthogonal codes for channel estimation and transmission [2], [11]. This results in, without loss of generality, the conditions

$$
\begin{aligned}
\mathbf{C}_{p} \mathbf{C}_{p}^{H} & =\frac{L_{p}}{L} \mathbf{I}_{N}, \\
\mathbf{C C}^{H} & =\tilde{\mathbf{C}} \tilde{\mathbf{C}}^{H}=\mathbf{I}_{N} .
\end{aligned}
$$

Note that (40) also implies $L_{p} \geq N$ and $L \geq N$. In addition, we consider $L=N$, which implies the condition

$$
\mathbf{C}^{H} \mathbf{C}=\tilde{\mathbf{C}}^{H} \tilde{\mathbf{C}}=\mathbf{I}_{L} .
$$

Substituting (40) and (41) in (35), we get

$$
\mathbf{A}=\left[\begin{array}{cc}
\left(1+\frac{L}{L_{p}}+2 \frac{\Omega E_{s}}{N_{0}}\right) \mathbf{I}_{L} & \left(\mathbf{I}_{L}+\frac{L}{L_{p}} \tilde{\mathbf{C}}^{T} \mathbf{C}^{*}\right) \\
-\frac{\Omega E_{s}}{N_{0}}\left(\mathbf{C}^{T} \tilde{\mathbf{C}}^{*}+\tilde{\mathbf{C}}^{T} \mathbf{C}^{*}\right) & -\left(1+\frac{L}{L_{p}}\right) \mathbf{I}_{L}
\end{array}\right]
$$

To obtain the eigenvalues of $\mathbf{A}$ in (42), we look at the characteristic equation

$$
\operatorname{det}\left(\mathbf{A}-\lambda \mathbf{I}_{2 L}\right)=0 .
$$

Let $\nu_{1}, \ldots, \nu_{K}$ denote the $K$ distinct real positive eigenvalues of the positive definite Hermitian matrix

$$
(\mathbf{C}-\tilde{\mathbf{C}})^{T}(\mathbf{C}-\tilde{\mathbf{C}})^{*}=2 \mathbf{I}_{L}-\left(\mathbf{C}^{T} \tilde{\mathbf{C}}^{*}+\tilde{\mathbf{C}}^{T} \mathbf{C}^{*}\right)
$$

such that $\nu_{i}$ has multiplicity $q_{i}$ for $i=1, \ldots, K$. Thus, $q_{1}+\cdots+$ $q_{K}=L$. Defining $\Gamma$, the average SNR per diversity branch, as

$$
\Gamma \triangleq \frac{\Omega E_{s}}{N_{0}}
$$


where $\Omega$ is the variance of the complex channel gain $h_{i j}$, we can simplify the characteristic equation after some algebra, resulting in

$$
\prod_{i=1}^{K}\left(\lambda^{2}-\Gamma \nu_{i} \lambda-\left(\frac{L}{L_{p}}+\left[1+\frac{L}{L_{p}}\right] \Gamma\right) \nu_{i}\right)^{q_{i}}=0
$$

Both the roots of

$$
\lambda^{2}-\Gamma \nu_{i} \lambda-\left(\frac{L}{L_{p}}+\left[1+\frac{L}{L_{p}}\right] \Gamma\right) \nu_{i}=0
$$

which we denote as $\lambda_{i 1}$ and $\lambda_{i 2}$, are real, one being positive and the other negative. Let $\lambda_{i 1}>0$ and $\lambda_{i 2}<0$. We can rewrite (36) as

$$
\Psi_{\mathcal{D}}(\jmath \omega)=\frac{1}{\prod_{i=1}^{K}\left[\left(1-2 \jmath \omega N_{0} \lambda_{i 1}\right)^{M q_{i}}\left(1-2 \jmath \omega N_{0} \lambda_{i 2}\right)^{M q_{i}}\right]}
$$

where

$$
\begin{aligned}
\lambda_{i 1}= & \frac{\Gamma \nu_{i}+\sqrt{\Gamma^{2} \nu_{i}^{2}+4\left(\frac{L}{L_{p}}+\left[1+\frac{L}{L_{p}}\right] \Gamma\right) \nu_{i}}}{2} \\
\lambda_{i 2}= & \frac{\Gamma \nu_{i}-\sqrt{\Gamma^{2} \nu_{i}^{2}+4\left(\frac{L}{L_{p}}+\left[1+\frac{L}{L_{p}}\right] \Gamma\right) \nu_{i}}}{2} .
\end{aligned}
$$

The poles of $\left(\Psi_{\mathcal{D}}\left(z /\left(2 N_{0}\right)\right)\right) / z$ which are on the left-half $z$-plane are $\lambda_{i 2}^{-1} i=1, \ldots, K$.

Define the functions $G_{j}(z)$ and $F_{j}(z)$ as

$$
\begin{aligned}
G_{j}(z) & \triangleq\left(z-\lambda_{j 2}^{-1}\right)^{M q_{j}} \frac{\Psi_{\mathcal{D}}\left(\frac{z}{2 N_{0}}\right)}{z} \\
F_{j}(z) & \triangleq \ln G_{j}(z) .
\end{aligned}
$$

From (39), the PEP is given by

$$
P(\mathbf{C} \rightarrow \tilde{\mathbf{C}})=-\sum_{j=1}^{K} \frac{1}{\left(M q_{j}-1\right) !} G_{j}^{\left(M q_{j}-1\right)}\left(\lambda_{j 2}^{-1}\right)
$$

where $G_{j}^{\left(M q_{j}-1\right)}\left(\lambda_{j 2}^{-1}\right)$ denotes the $\left(M q_{j}-1\right)$ th derivative of $G_{j}(z)$ evaluated at $z=\lambda_{j 2}^{-1}$.
Now the $m$ th derivative of $F_{j}(z)$ can be expressed as

$$
\begin{aligned}
F_{j}^{(m)}(z)= & \frac{(-1)^{m}(m-1) !}{z^{m}} \\
& +(m-1) ! M \sum_{i=1}^{K} \frac{q_{i} \lambda_{i 1}^{m}}{\left(1-z \lambda_{i 1}\right)^{m}} \\
& +(m-1) ! M \sum_{\substack{i=1 \\
i \neq j}}^{K} \frac{q_{i} \lambda_{i 2}^{m}}{\left(1-z \lambda_{i 2}\right)^{m}}
\end{aligned}
$$

Using Faa di Bruno's formula [10], the $\left(M q_{j}-1\right)$ th derivative of $G_{j}(z)$ can be expressed as

$$
\begin{aligned}
& G_{j}^{\left(M q_{j}-1\right)}(z)=\left(M q_{j}-1\right) ! G_{j}(z) \\
& \times \sum_{\substack{\left(l_{1}, \ldots, l_{M q_{j}-1}\right) \\
0 \leq l_{1}, \ldots, l_{M q_{j}-1 \leq M q_{j}-1} \\
l_{1}+2 l_{2}+\cdots+\left(M q_{j}-1\right) l_{M q_{j}-1}=M q_{j}-1}} \prod_{m=1}^{M q_{j}-1} \frac{1}{l_{m} !} \\
& \times\left(\frac{F_{j}^{(m)}(z)}{m !}\right)^{l_{m}}
\end{aligned}
$$

where the summation is over all $\left(M q_{j}-1\right)$-tuples $\left(l_{1}, \ldots, l_{M q_{j}-1}\right)$ of integers in the range $\left[0, M q_{j}-1\right]$ satisfying $\sum_{m=1}^{M q_{j}-1} m l_{m}=M q_{j}-1$. Substituting (51) and (50) in (49), we obtain (52), as found at the bottom of the page,where $\left\{\lambda_{i 1}\right\},\left\{\lambda_{i 2}\right\}$ are given by (47). Thus, (52) is a closed-form expression for the PEP in terms of the distinct real positive eigenvalues $\nu_{1}, \ldots, \nu_{K}$ of the matrix $(\mathbf{C}-\tilde{\mathbf{C}})^{T}(\mathbf{C}-\tilde{\mathbf{C}})^{*}$.

To numerically compute the PEP from this expression, we need a precalculated lookup table of enumerations of the indexes $l_{1}, l_{2}, \ldots$, in the composite summation of (52) and the eigenvalues $\nu_{1}, \ldots, \nu_{K}$. The lookup table generation, the eigenvalue computation, and the subsequent simple operations which yield the PEP can be easily performed using a mathematical software like MATLAB.

Let the number of enumerations of $\left(l_{1}, \ldots, l_{M q_{j}-1}\right)$ for which the equality

$$
l_{1}+2 l_{2}+\cdots+\left(M q_{j}-1\right) l_{M q_{j}-1}=M q_{j}-1
$$

holds when $0 \leq l_{1}, \ldots, l_{M q_{j}-1} \leq M q_{j}-1$ be denoted as a function $f\left(M q_{j}\right)$. For given $L, M$, and $q_{1}, \ldots, q_{K}$, the total number of summations in (52), denoted as $\mathcal{S}_{L, M}$, is then given by

$$
\mathcal{S}_{L, M}=f\left(M q_{1}\right)+\cdots+f\left(M q_{K}\right)
$$

$$
\begin{aligned}
& P(\mathbf{C} \rightarrow \tilde{\mathbf{C}}) \\
& =\sum_{j=1}^{K} \frac{\left(-\lambda_{j 2}\right)^{M\left(2 L-q_{j}\right)}}{\left[\prod_{i=1}^{K}\left(\lambda_{i 1}-\lambda_{j 2}\right)^{M q_{i}}\right]\left[\prod_{\substack{i=1 \\
i \neq j}}^{K}\left(\lambda_{i 2}-\lambda_{j 2}\right)^{M q_{i}}\right]} \\
& \quad \times \sum_{\substack{\left(l_{1}, \ldots, l_{M q_{j}-1}\right) \\
0 \leq l_{1}, \ldots, l_{M q_{j}-1 \leq M q_{j}-1}}}^{M q_{j}-1} \prod_{m=1}^{1} \frac{1}{l_{m} !}\left[\frac{M}{m} \sum_{i=1}^{K} \frac{q_{i} \lambda_{i 1}^{m}}{\left(\lambda_{i 1}-\lambda_{j 2}\right)^{m}}+\frac{M}{m} \sum_{\substack{i=1 \\
i \neq j}}^{K} \frac{q_{i} \lambda_{i 2}^{m}}{\left(\lambda_{i 2}-\lambda_{j 2}\right)^{m}}\right]^{l_{m}}
\end{aligned}
$$


TABLE I

NUMBER OF ENUMERATIONS OF $l_{1}, \ldots, l_{M q_{j}-1}$ IN (52)

\begin{tabular}{c|c}
\hline$M q_{j}$ & $f\left(M q_{j}\right)$ \\
\hline \hline 2 & 1 \\
\hline 3 & 2 \\
\hline 4 & 3 \\
\hline 5 & 5 \\
\hline 6 & 7 \\
\hline 7 & 11 \\
\hline 8 & 15 \\
\hline 9 & 22 \\
\hline 10 & 30 \\
\hline 11 & 42 \\
\hline 12 & 56 \\
\hline 13 & 77 \\
\hline 14 & 101 \\
\hline 15 & 135 \\
\hline 16 & 176 \\
\hline &
\end{tabular}

This number of summations is bounded by two extremes. At the lower bound, we have the case

$$
K=L, \quad q_{1}=\cdots=q_{L}=1
$$

while at the upper bound we have the case

$$
K=1, \quad q_{1}=L
$$

Therefore, from (53), we can write

$$
L f(M) \leq \mathcal{S}_{L, M} \leq f(M L) .
$$

Values of $f\left(M q_{j}\right)$ for $M q_{j}=2, \ldots, 16$ are shown in Table I. Table II shows the lower and upper bounds on the total number $S_{L, M}$ of summations in (52) for some typical values of $L$ and $M$.

When the channel estimate is perfect, we have $\hat{\mathbf{H}}=\mathbf{H}$ in (13), implying $\mathbf{B}=\tilde{\mathbf{B}}=\mathbf{0}_{L_{p} \times L}$ in the matrix $\mathbf{A}$ given by (35). This also implies the condition $L_{p} \gg L$. By putting $L / L_{p}=0$ in (45), the characteristic equation of $\mathbf{A}$ can now be written as

$$
\prod_{i=1}^{K}\left(\lambda^{2}-\Gamma \nu_{i} \lambda-\Gamma \nu_{i}\right)^{q_{i}}=0 .
$$

Let $\lambda_{i 1}^{\prime}$ and $\lambda_{i 2}^{\prime}$ be the roots of $\lambda^{2}-\Gamma \nu_{i} \lambda-\Gamma \nu_{i}=0$, where $\Gamma$ is defined in (44), with $\lambda_{i 1}^{\prime}>0$ and $\lambda_{i 2}^{\prime}<0$. Thus

$$
\lambda_{i 1}^{\prime}, \lambda_{i 2}^{\prime}=\frac{\Gamma \nu_{i} \pm \sqrt{\Gamma^{2} \nu_{i}^{2}+4 \Gamma \nu_{i}}}{2} .
$$

Therefore, the PEP for perfect channel estimation is given by (52) with $\lambda_{i 1}$ replaced by $\lambda_{i 1}^{\prime}$ and $\lambda_{i 2}$ by $\lambda_{i 2}^{\prime}$.
TABLE II

LOWER AND UPPER BOUNDS ON THE TOTAL NUMBER $\mathcal{S}_{L, M}$ OF SUMMATIONS IN (52)

\begin{tabular}{c|c|c}
\hline$(L, M)$ & $\begin{array}{c}\text { Lower Bound } \\
L f(M)\end{array}$ & $\begin{array}{c}\text { Upper Bound } \\
f(M L)\end{array}$ \\
\hline \hline$(2,2)$ & 2 & 3 \\
\hline$(2,3)$ & 4 & 7 \\
\hline$(2,4)$ & 6 & 15 \\
\hline \hline$(3,2)$ & 3 & 7 \\
\hline$(3,3)$ & 6 & 22 \\
\hline$(3,4)$ & 9 & 56 \\
\hline \hline$(4,2)$ & 4 & 15 \\
\hline$(4,3)$ & 8 & 56 \\
\hline$(4,4)$ & 12 & 176 \\
\hline
\end{tabular}

\section{AN EXAMPLE}

Consider a system using the Alamouti code [2] with $L=$ $N=L_{p}=2$. A codeword is given by

$$
\mathbf{C}=\left[\begin{array}{cc}
s_{1} & -s_{2}^{*} \\
s_{2} & s_{1}^{*}
\end{array}\right] .
$$

Take the case of BPSK, where $s_{1}, s_{2} \in\{-1 / \sqrt{2}, 1 / \sqrt{2}\}$. We have four possible code matrices. Assuming all code matrices are equally likely, the matrix $(\mathbf{C}-\tilde{\mathbf{C}})^{T}(\mathbf{C}-\tilde{\mathbf{C}})^{*}$ takes the values $2 \mathbf{I}_{2}$ with probability $2 / 3$ and $4 \mathbf{I}_{2}$ with probability $1 / 3$. Thus, we have either $\nu_{1}=\nu_{2}=2$ or $\nu_{1}=\nu_{2}=4$. Therefore, $K=1$ and $q_{1}=2$. Denoting $\nu=\nu_{1}=\nu_{2}, \lambda_{1}=\lambda_{11}$, and $\lambda_{2}=\lambda_{12}$, we get from (52) the PEP

$$
\begin{aligned}
P & (\mathbf{C} \rightarrow \tilde{\mathbf{C}}) \\
= & P(\nu, \Gamma) \\
= & \frac{\left(-\lambda_{2}\right)^{2 M}}{\left(\lambda_{1}-\lambda_{2}\right)^{2 M}} \\
& \times \sum_{\substack{\left.0 \leq l_{1}, \ldots, l_{2 M-1} \leq 2 M-1 \\
l_{2}, l_{2 M-1}\right)}}^{l_{1}+2 l_{2}+\cdots+(2 M-1) l_{2 M-1}=2 M-1} \\
& \times\left[\frac{1}{m}+\frac{2 M}{m} \frac{\lambda_{1}^{m}}{\left(\lambda_{1}-\lambda_{2}\right)^{m}}\right]^{l_{m}}
\end{aligned}
$$

where, from (47)

$$
\lambda_{1}, \lambda_{2}=\frac{\Gamma \nu \pm \sqrt{\Gamma^{2} \nu^{2}+4(1+2 \Gamma) \nu}}{2} .
$$

We can replace $\lambda_{1}, \lambda_{2}$ in (58) by $\lambda_{1}^{\prime}, \lambda_{2}^{\prime}$, respectively, to get the PEP with perfect channel estimation. Note from (56) that

$$
\lambda_{1}^{\prime}, \lambda_{2}^{\prime}=\frac{\Gamma \nu \pm \sqrt{\Gamma^{2} \nu^{2}+4 \Gamma \nu}}{2} .
$$

The average PEP is given by

$$
P(\Gamma)=(2 / 3) P(2, \Gamma)+(1 / 3) P(4, \Gamma) .
$$




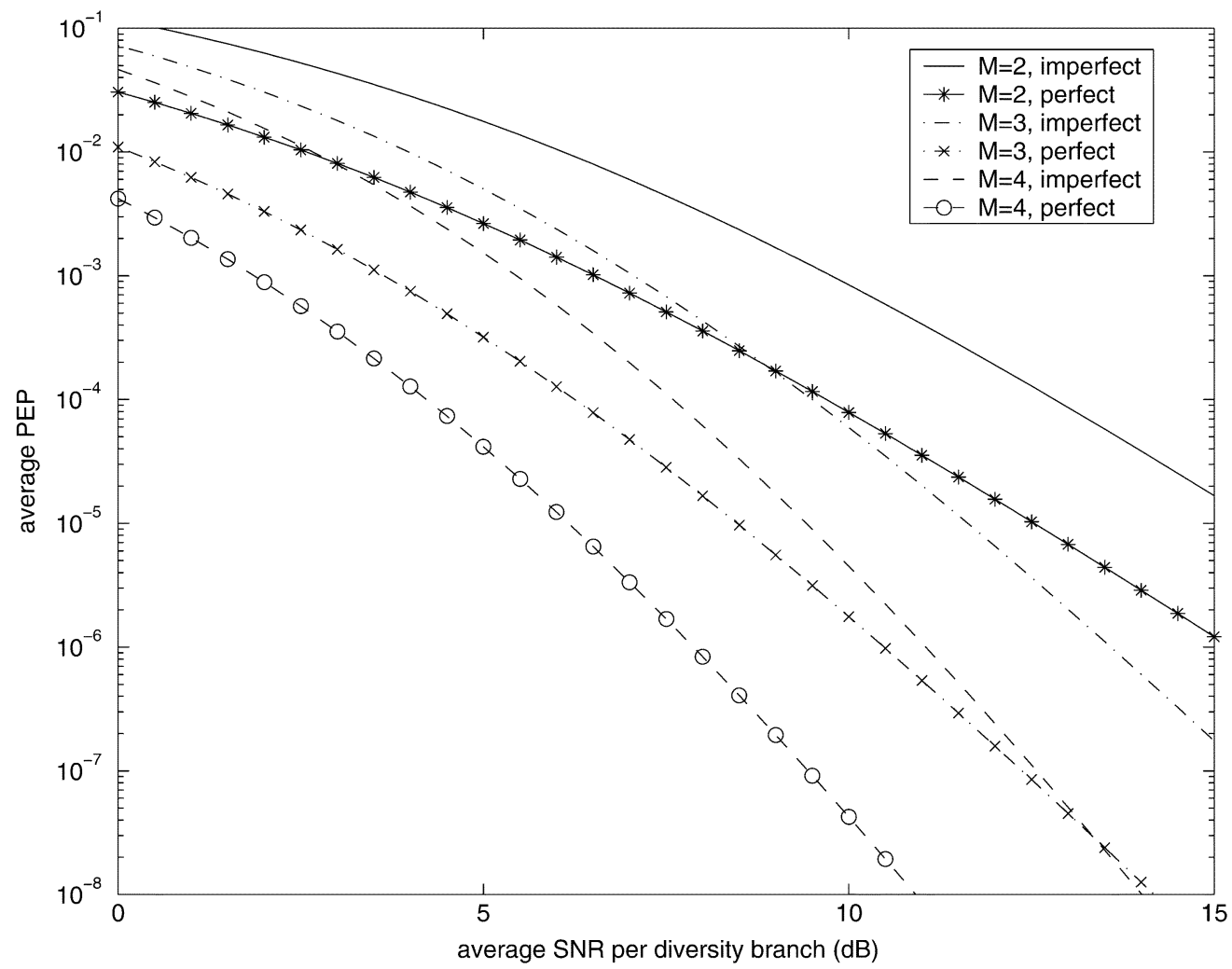

Fig. 1. Average PEP versus $\Gamma$ for the Alamouti code with BPSK, 2 transmit antennas $(N=2), 2$ symbol intervals $(L=2), 2$ pilot code vectors $\left(L_{p}=2\right)$, and number of receive antennas $M=2,3,4$.

(a) $M=2$

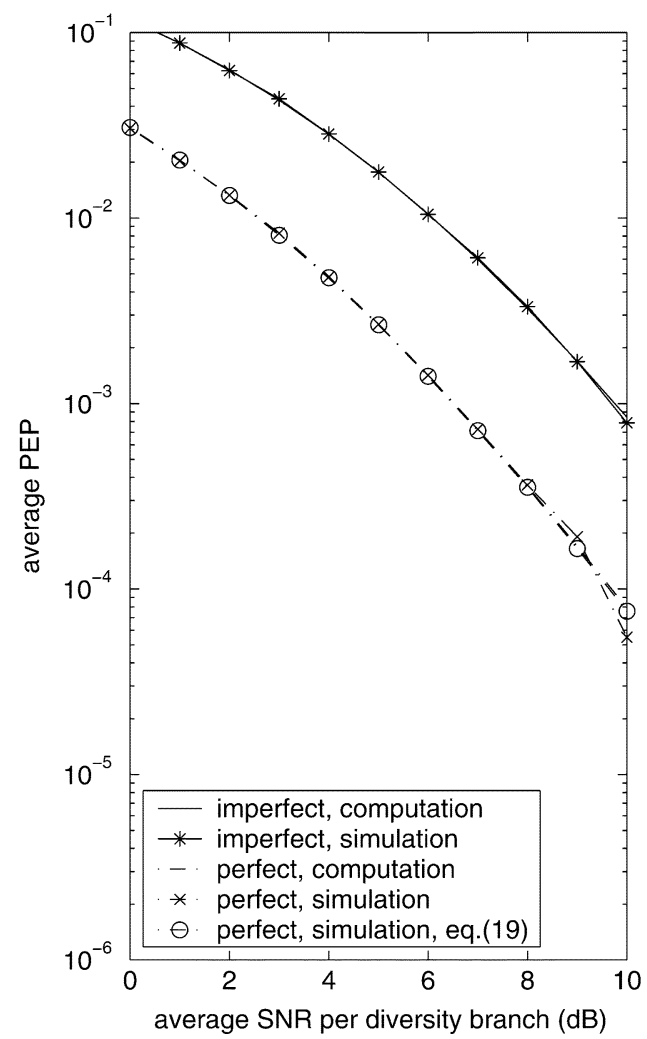

(b) $M=3$

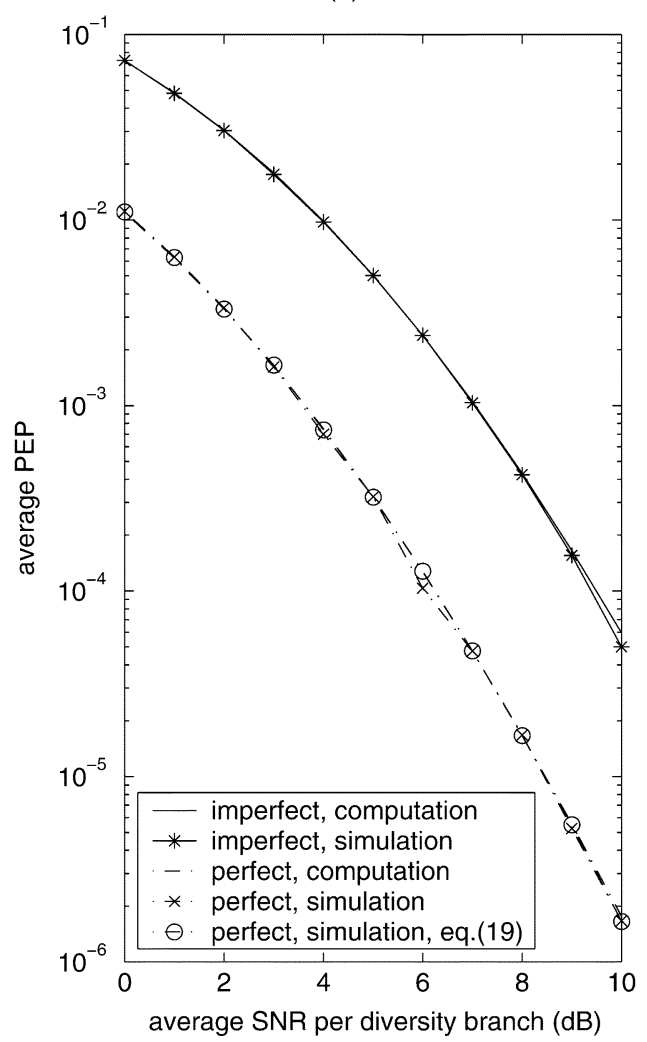

Fig. 2. Comparison of computed and simulated average PEP versus $\Gamma$ for the Alamouti code with BPSK, 2 transmit antennas $(N=2), 2$ symbol intervals $(L=2), 2$ pilot code vectors $\left(L_{p}=2\right)$, and number of receive antennas $M=2,3$.

Plots of the average PEP both with imperfect and perfect channel estimation, computed using (58) and (61), are shown in Fig. 1. We find that the degradation in performance due to imperfect channel estimation can be compensated by increasing 


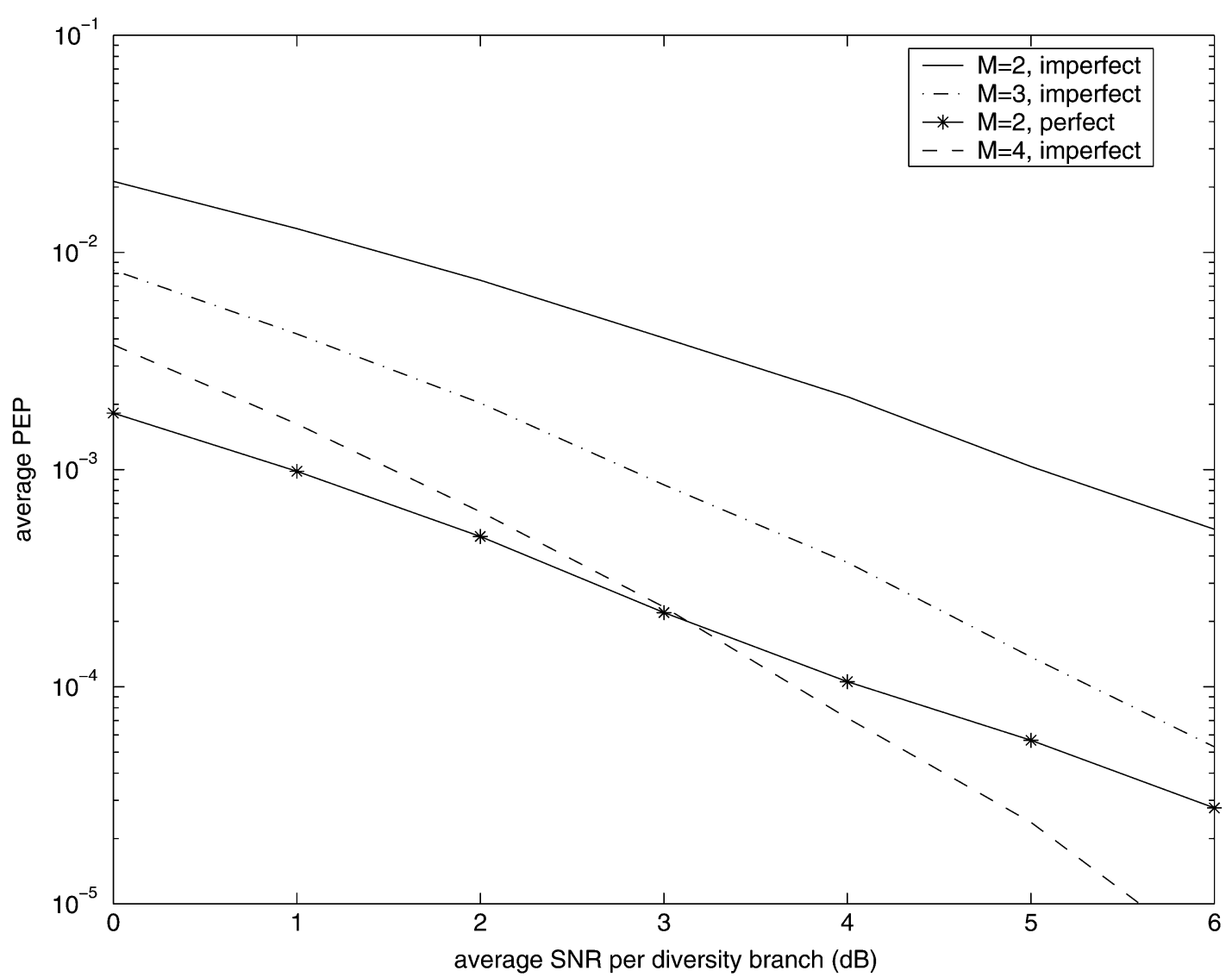

Fig. 3. Average PEP obtained by simulation versus $\Gamma$ for a nonorthogonal space-time code with BPSK, 3 transmit antennas $(N=3)$, 3 symbol intervals $(L=3)$, and 3 pilot code vectors $\left(L_{p}=3\right)$.

the number $M$ of receive antennas. For example, at $\Gamma \approx 9 \mathrm{~dB}$, the average PEP with $M=3$ and imperfect channel estimation is the same as that with $M=2$ and perfect channel estimation.

Comparison of the average PEP obtained by computation using (58) and (61) with that obtained by simulation is shown in Fig. 2(a) for $M=2$ and in Fig. 2(b) for $M=3$. In case of perfect channel estimation, the simulation results obtained by averaging (19) over the statistics of $\mathbf{H}$ are also included. The simulation results are found to match closely with the computed results. In case of imperfect channel estimation, it is not possible to obtain a characterization of the PEP similar to (19).

Plots of the average PEP obtained by simulation for a nonorthogonal space-time code with $L=N=L_{p}=3$, is shown in Fig. 3. Here a codeword is given by [12]

$$
\mathbf{C}=\left[\begin{array}{ccc}
s_{1} & s_{2}^{*} & -s_{3}^{*} \\
s_{2} & -s_{1}^{*} & -s_{3}^{*} \\
s_{3} & s_{1} & s_{2}^{*}
\end{array}\right] .
$$

We again take the case of BPSK with $s_{1}, s_{2}, s_{3} \in\{-1,1\}$. It is found that an increase in the number of receive antennas can compensate for the degradation in performance due to imperfect channel estimation. For example, at $\Gamma \approx 3 \mathrm{~dB}$, using 4 receive antennas with imperfect channel estimation can achieve the same average PEP as with 2 receive antennas and perfect channel estimation. This behavior is the same as that seen in Fig. 1 with the Alamouti code, which is an orthogonal space-time code.

\section{CONCLUSION}

We have analyzed the error performance of a space-time coding system with imperfect channel estimation in flat Rayleigh fading. Using the cf. of the decision variable, we have derived a closed-form expression for the PEP. The same expression can also be used to obtain the PEP in the case of perfect channel estimation. We have considered an example of a system using the Alamouti code. Numerical results show the degradation in performance due to imperfect channel estimation that can be compensated by increasing the number of receive antennas.

\section{REFERENCES}

[1] V. Tarokh, N. Sheshadri, and A. R. Calderbank, "Space-time codes for high data rate wireless communications: Performance criteria and code construction," IEEE Trans. Inf. Theory, vol. 44, pp. 744-765, Mar. 1998.

[2] S. M. Alamouti, "A simple transmitter diversity scheme for wireless communications," IEEE J. Sel. Areas Commun., vol. 16, pp. 1451-1458, Oct. 1998.

[3] H. Arslan and G. E. Bottomley, "Channel estimation in narrowband wireless communication systems," Wireless Commun. Mobile Comput., vol. 1, pp. 201-219, Apr.-Jun. 2001.

[4] Y. G. Li, N. Sheshadri, and S. Ariyavisitakul, "Channel estimation for OFDM systems with transmitter diversity in mobile wireless channels," IEEE J. Sel. Areas Commun., vol. 17, pp. 461-471, Mar. 1999.

[5] Y. G. Li, "Simplified channel estimation for OFDM system with multiple transmit antennas," IEEE Trans. Wireless Commun., vol. 1, pp. 67-75, Jan. 2002.

[6] A. F. Naguib, V. Tarokh, N. Sheshadri, and A. R. Calderbank, "A spacetime coding modem for high-data-rate wireless communications," IEEE J. Sel. Areas Commun., vol. 16, pp. 1459-1478, Oct. 1998 
[7] G. Taricco and E. Biglieri, "Exact pairwise error probability of spacetime codes," IEEE Trans. Inf. Theory, vol. 48, pp. 510-513, Feb. 2002.

[8] G. L. Turin, "The characteristic function of Hermitian quadratic forms in complex normal variables," Biometrika, vol. 47, pp. 199-201, 1960.

[9] J. Gil-Pelaez, "Note on the inversion theorem," Biometrika, vol. 38, pp. 481-482, 1951

[10] C.-J. de la Vallee Poussin, Cours D'Analyze Infinitesimale, 12th ed, Paris, France: Gauthier-Villars, Libraire Universitaire Louvain, 1959, vol. 1.

[11] V. Tarokh, H. Jafarkhani, and A. R. Calderbank, "Space-time block codes from orthogonal designs," IEEE Trans. Inf. Theory, vol. 45, pp. 1456-1467, Jul. 1999.

[12] M. Uysal and C. N. Georghiades, "Non-orthogonal space-time block codes for 3TX antennas," Electron. Lett., vol. 38, no. 25, pp. 1689-1691, Dec. 5, 2002.

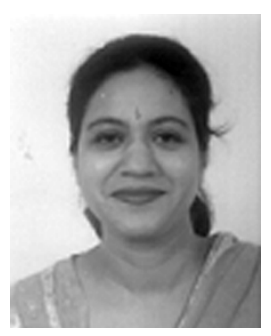

Parul Garg was born in Ghaziabad, India, on September 25, 1968. She received the B.Sc. Engg. and M.Sc. Engg. degrees in electronics engineering from Aligarh Muslim University, Aligarh, India, in 1990 and 1994, respectively. She is currently pursuing the $\mathrm{Ph} . \mathrm{D}$. degree in electrical engineering at the Indian Institute of Technology, Delhi.

From May 1996 to July 2000, she worked as a faculty member at the Institute of Engineering and Technology, Lucknow, India. Since July 2000, she has been working as a faculty member at the Netaji Subhas Institute of Technology, New Delhi, India. Her current work mainly focuses on different aspects of wireless communications with emphasis on channel estimation and diversity techniques.

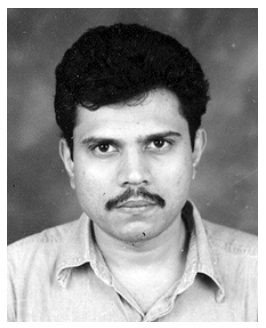

Ranjan K. Mallik (S'88-M'93-SM'02) was born in Calcutta, India, on November 15, 1964. He received the B.Tech. degree in electrical engineering from the Indian Institute of Technology, Kanpur, in 1987 and the M.S. and Ph.D. degrees in electrical engineering from the University of Southern California, Los Angeles, in 1988 and 1992, respectively.

From August 1992 to November 1994, he was a scientist at the Defence Electronics Research Laboratory, Hyderabad, India, working on missile and EW projects. From November 1994 to January 1996, he was a faculty member of the Department of Electronics and Electrical Communication Engineering, Indian Institute of Technology, Kharagpur. In January 1996, he joined the faculty of the Department of Electronics and Communication Engineering, Indian Institute of Technology, Guwahati, where he worked until December 1998. Since December 1998, he has been with the Department of Electrical Engineering, Indian Institute of Technology, Delhi, where he is an Associate Professor. His research interests are in communication theory and systems, difference equations, and linear algebra.

Dr. Mallik is a member of Eta Kappa Nu. He is also a Member of the American Mathematical Society, the International Linear Algebra Society, the Indian National Academy of Engineering and The Institution of Electrical Engineers, a Fellow of The Institution of Electronics and Telecommunication Engineers, a Life Member of the Indian Society for Technical Education, and an Associate Member of The Institution of Engineers (India).

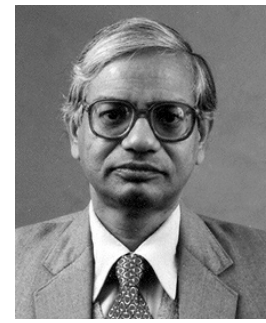

Hari M. Gupta received the B.E. degree from the University of Roorkee, now the Indian Institute of Technology (IIT), Roorkee, in 1967, the M.Tech. degree from IIT Kharagpur in 1969, and the Ph.D. degree from IIT Kanpur in 1974.

He joined the faculty of the Department of Electrical Engineering at IIT Delhi in 1973 where he has been a Professor since 1986. He has been the Dean of Undergraduate Studies, the Head of the Department of Electrical Engineering, and the Coordinator of the Bharti School of Telecommunication Technology and Management at IIT Delhi. He has held faculty appointments at McGill University, Montreal, Canada, and Drexel University, Philadelphia, PA. He has been an academic visitor to the University of Maryland, College Park, MD, Media Lab. at the Massachusetts Institute of Technology, Cambridge, MA, the Swiss Federal Institute of Technology, Lausanne, Switzerland, and several British universities. His academic and professional interests are in telecommunication systems, computer communication networks, multimedia systems, and photonic information systems.

Dr. Gupta has been India's representative on the International Federation for Information Processing (IFIP) Communications Committee. He is a Fellow of The Institution of Electronics and Telecommunication Engineers (IETE) and The Institution of Engineers (India), and a Senior Member of the Computer Society of India (CSI). He has been the Vice-President of the Systems Society of India, a Council Member of IETE, the Chairman of the Data Communication Division of CSI, and a Founder Member of the Association for Security of Information Systems (ASIS). 